\title{
Co-cultures of renal progenitors and endothelial cells on kidney decellularized matrices replicate the renal tubular environment in vitro
}

\author{
Rita Sobreiro-Almeida $^{1,2}$ (D) ｜ Maria Elena Melica ${ }^{3,4}$ (D) | Laura Lasagni ${ }^{3,4}$ (iD | \\ Paola Romagnani $^{3,4,5}$ (D) | Nuno M. Neves ${ }^{1,2}$ (i)
}

13B's Research Group, I3Bs - Research Institute on Biomaterials, Biodegradables and Biomimetics, University of Minho, Headquarters of the European Institute of Excellence on Tissue Engineering and Regenerative Medicine, Barco, Portugal

${ }^{2}$ ICVS/3B's - PT Government Associate Laboratory, Braga/Guimarães, Portugal

${ }^{3}$ Department of Clinical and Experimental Biomedical Sciences "Mario Serio", University of Florence, Florence, Italy

${ }^{4}$ Excellence Centre for Research, Transfer and High Education for the Development of DE NOVO Therapies, Florence, Italy

${ }^{5}$ Nephrology and Dialysis Unit, Meyer Children's University Hospital, Florence, Italy

\section{Correspondence}

Nuno M. Neves, 3B's Research Group, I3Bs - Research Institute on Biomaterials, Biodegradables and Biomimetics, University of Minho, Headquarters of the European Institute of Excellence on Tissue Engineering and Regenerative Medicine, AvePark, Parque de Ciência e Tecnologia, Zona Industrial da Gandra, Barco 4805-

017, Guimarães, Portugal.

Email: nuno@i3bs.uminho.pt

Funding information

Fundação para a Ciência e a

Tecnologia, Grant/Award Number: PD/ BD/128102/2016; European Regional Development Fund, Grant/Award Number: NORTE-01-0145-FEDER-000023

\begin{abstract}
Aim: Herein we propose creating a bilayer tubular kidney in-vitro model. It is hypothesized that membranes composed of decellularized porcine kidney extracellular matrix are valid substitutes of the tubular basement membrane by mimicking the physiological relevance of the in vivo environment and disease phenotypes.

Methods: Extracellular matrix was obtained from decellularized porcine kidneys. After processing by lyophilization and milling, it was dissolved in an organic solvent and blended with poly(caprolactone). Porous membranes were obtained by electrospinning and seeded with human primary renal progenitor cells to evaluate phenotypic alterations. To create a bilayer model of the in vivo tubule, the same cells were differentiated into epithelial tubular cells and co-cultured with endothelial cells in opposite sites.
\end{abstract}

Results: Our results demonstrate increasing metabolic activity, proliferation and total protein content of renal progenitors over time. We confirmed the expression of several genes encoding epithelial transport proteins and we could also detect tubularspecific proteins by immunofluorescence stainings. Functional and transport assays were performed trough the bilayer by quantifying both human serum albumin uptake and inulin leakage. Furthermore, we validated the chemical modulation of nephrotoxicity on this epithelium-endothelium model by cisplatin exposure.

Conclusion: The use of decellularized matrices in combination with primary renal cells was shown to be a valuable tool for modelling renal function and disease in vitro. We successfully validated our hypothesis by replicating the physiological conditions of an in vitro tubular bilayer model. The developed system may contribute significantly for the future investigation of advanced therapies for kidney diseases.

\section{K E Y W O R D S}

cellular crosstalk, decellularization, extracellular matrix, kidney, renal progenitors, tubule 


\section{INTRODUCTION}

The human kidney filters nearly $2 \mathrm{~L}$ of primary urine from plasma every day, in which millions of nephrons work together to regulate the complex process of filtration. As part of the nephron unit, the tubules are mainly responsible to maintain normal physiological homeostasis and electrolyte balance by regulating the volume and reabsorption of solutes. ${ }^{1}$ The epithelial cells that compose the cellular barrier of the tubule express several ion and water channels as well as transporters which can modify the composition of the filtrate according to the physiological needs. ${ }^{2}$ This vital function makes the tubules highly susceptible to damage induced from drugs, toxins and blood-borne diseases, which frequently lead to cell death and tubular dysfunction.,

The ability to develop in vitro models that can recapitulate the renal tubular environment would be of great interest for therapeutic development and discovery but also to understand mechanisms underlying kidney pathology and homeostasis. Tubular cells are attached to an underlying basement membrane (BM), composed by typical elements of the extracellular matrix (ECM), which when dysregulated can affect cell attachment, proliferation, differentiation and migration. Indeed, changes on the composition and thickness of the BM are linked with pathological conditions, such as inflammatory processes, renal fibrosis and epithelial-to-mesenchymal transitions. ${ }^{5}$ Major efforts have been accomplished in this area, namely, the creation of functional kidney microtissues, microphysiological fluidic kidney systems ${ }^{7,8}$ or even strategies to mimic the organic cation transport. ${ }^{9}$ Although these systems were developed with the main aim of preclinical screening, they are far from replicating the in vivo contact with the BM and the cellular crosstalk between endothelial and epithelial cells, both crucial factors on the filtration process of the kidney. ${ }^{1}$ The endothelium and/or peritubular capillaries are in close proximity to the tubular epithelium, not only when considering physiological processes but also under pathophysiological conditions. ${ }^{10}$ Several studies have demonstrated the importance of the endothelium for a normal kidney function. A complex communication network between epithelial cells composing the tubule and endothelial cells of the vasculature has already been demonstrated to influence solute transport, angiogenesis and enriched expression of specific genes. ${ }^{10-13}$ These co-culture systems proved not only the importance of having endothelial cells on an in vitro model but also the importance of recapitulating the BM permeability and constitution. Although in the past few years huge advances were reported in co-culture systems, their standardization is still difficult. Not only these systems face the problem of epithelial dedifferentiation but also they are composed by synthetic materials which frequently fail to reproduce the in vivo structure and function, being the majority of times coated with different ECM proteins. ${ }^{14,15}$
Previously, we proposed the use of electrospun membranes combining poly(caprolactone) (PCL) and decellularized porcine kidney ECM (1:1) as valuable replicas of renal basement membranes. We have demonstrated their value by comparing proximal tubular cellular performance on these meshes with bare PCL electrospun meshes. ${ }^{16}$ Herein, we propose a study focused on the cellular performance with renal progenitor cells and a second study focused on establishing an in vitro bilayer model to further validate our initial hypothesis. We hypothesize that this membrane would be extremely valuable to recapitulate the physiological environment, in such a way that would provide cues to renal progenitor cells (hRPCs) to differentiate and form a functional epithelial monolayer. These cells represent a subset of multipotent progenitors present in the Bowman's capsule, which can be purified from cultured capsulated glomeruli. They were already shown to differentiate into several lineages including osteogenic cells, adipocytes and cells that exhibit phenotypic and functional features of neuronal cells. ${ }^{17}$ Additionally, their differentiation potential was further extended for proximal and/or distal tubule cells and podocytes, upon culture with specific supplementation. ${ }^{18}$ Indeed, hRPCs were demonstrated to be involved in the regeneration of different portions of the nephron after injury. ${ }^{19}$ Accordingly, we hypothesize that we can achieve tubular differentiation from hRPCs without supplementation on our membranes. Additionally, we focused our study on demonstrating transport properties and induction of disease phenotypes on a bilayer model, composed of epithelial and endothelial cells, mimicking the organization of an in vivo tubule. Considering the findings that we have found, these models are proposed as valuable tools for integrating dialysis equipments or bioartificial kidneys in the future.

\section{2 | RESULTS}

\section{1 | dKECM and dKECM-derived membrane characterization}

To confirm that processing steps were not causing adverse effects on the protein content of kidney ECM, collagen content was assessed by immunofluorescence (IF) methods, being the major component of kidney ECM. ${ }^{20}$ Fibronectin was additionally assessed as a well-known protein which mediates cell-substratum adhesion. ${ }^{21}$ Preservation of collagen I, collagen IV and fibronectin was confirmed by green staining on the micrographs after sterilization process (Figure 1A-C respectively). No differences in fluorescence intensity were found between these micrographs and the ones obtained before sterilization process (data not shown), which confirms protein preservation after processing steps. Nuclear staining was also performed on IF sections by DAPI, although not visible on the micrographs, confirming effective removal of 

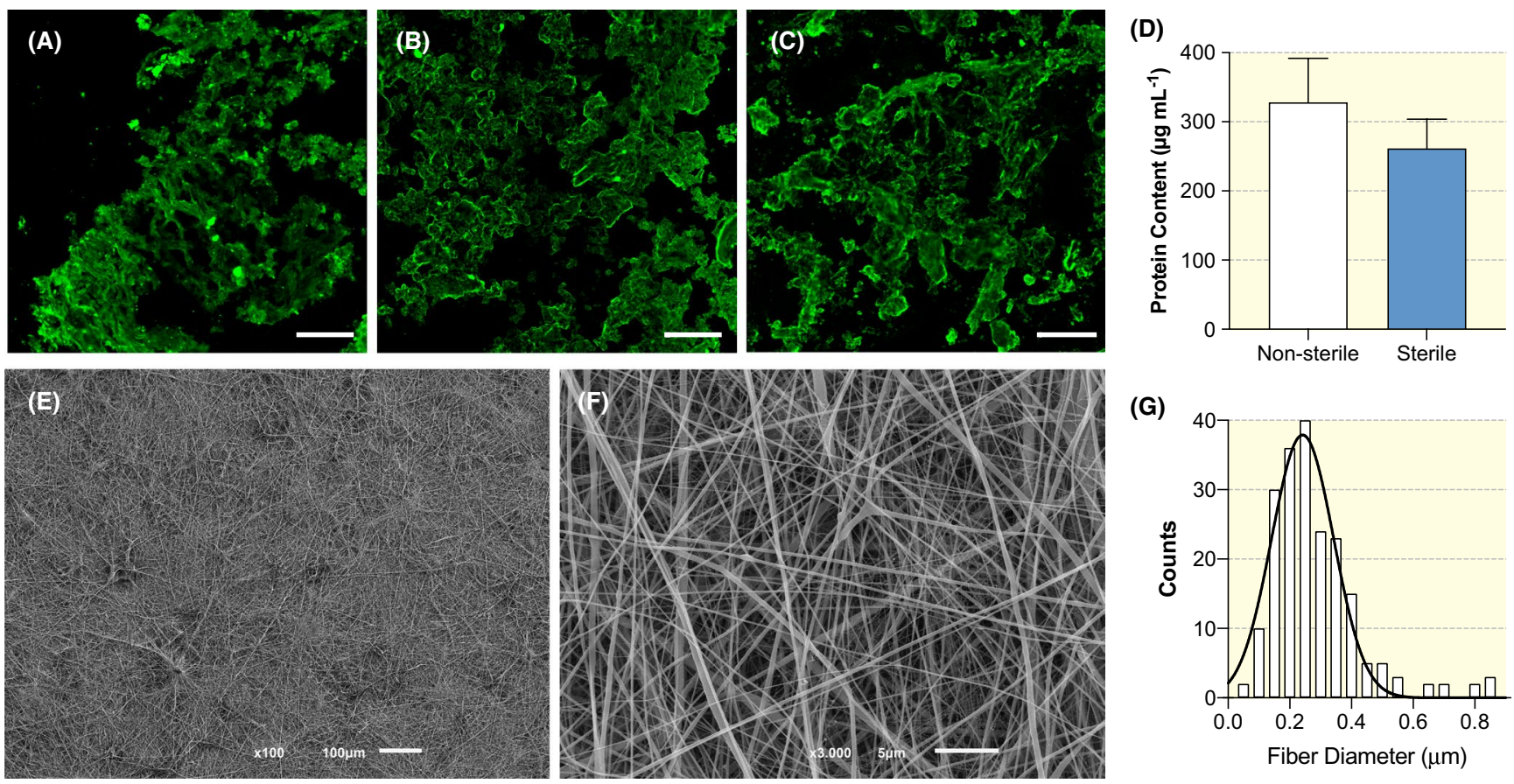

F I G U R E 1 dKECM characterization. (A-C) Immunofluorescence of ECM-related proteins ( green) on cryosections of decellularized powder: (A) Collagen type I, (B) Collagen type IV and (C) Fibronectin; (D) Protein quantification of decellularized powder demonstrates preservation of protein content after sterilization process; (E, F) SEM micrographs of the fibres at 100 and 3000x magnification respectively; (g) Fiber diameter distribution was measured from 3 random sites on 3 different batches of electrospun membranes. Fibres are randomly distributed and present an average diameter of $0.242 \mu \mathrm{m}$. Scale bars: (A-C and E) $100 \mu \mathrm{m}$, (F) $5 \mu \mathrm{m}$

cellular content. Moreover, biochemical quantification of total protein content was performed (Figure 1D) and the difference on the quantity of protein per mg was found no significant after sterilization (with a mean of $\sim 328$ and $~ 261 \mu \mathrm{g} /$ $\mathrm{mL}$ for non-sterile and sterile conditions respectively).

SEM micrographs of the electrospun meshes revealed a fibrous surface with randomly oriented fibres (Figure 1E,F). These micrographs reveal the nanostructure of these fibres, which present an average diameter of $0.242 \pm 0.1 \mu \mathrm{m}$, following a normal distribution (Figure 1G). These nanofibres will potentially aid on cellular adhesion without compromising cellular proliferation because of their large surface area. Thickness was measured from SEM micrographs by performing a cross section and placing the membrane at a $90^{\circ}$ angle. The measured thickness was $\sim 22 \pm 5.19 \mu \mathrm{m}$, which will allow for physical separation of hRPCs and endothelial cells, while maintaining the communication between the two cell types. Porosity measurements revealed the percentage of porous space existent in the membrane. We calculated a porosity value of $97 \pm 1.28 \%$, which allows for the selective passage of solutes. These models also require the maintenance of the ultrastructure and very low percentage of weight loss, which we tested during a 30-day degradation assay, where only 3\% of the mass was lost, proving that our membrane has a strong ECM crosslink and also a good blend between decellularized matrix and PCL. This enables performing long-term studies with the membrane being confident that its proprieties are not lost. Additionally, the membranes shown having a very high water uptake capacity of nearly $499 \%$, which further corroborates the high porosity results. The nanometer-scale fibres will support higher cell attachment, while providing a solid substrate with high porosity and water uptake, enabling solute, oxygen and growth factors passage between the two compartments of the co-culture system.

\section{2 | Cellular behaviour of hRPCs on decellularized matrix membranes}

Before all the experiments, hRPCs were characterized for simultaneous expression of CD133 and CD24 by flow cytometry. hRPCs between passages 1-3 from two different donors were used. All of the populations presented double positivity between $90 \%$ and $95 \%$, a signature preserved until passage 3 . In this work, the construction of the bilayer model started with a simplistic study on our dKECM membranes where hRPCs growth and behaviour was characterized in a culture time of 21 days. For that it was necessary to physically separate the HUVECs from hRPCs. Figure 2 represents the results for cellular metabolic activity (by alamarBlue assay-Figure 2A) and proliferation (by quantification of DNA content-Figure 2B) for mono- and co-cultures. hRPCs were shown viable and proliferating in all time points when in contact with dKECM, with increasing values over time. Interestingly, we observed 

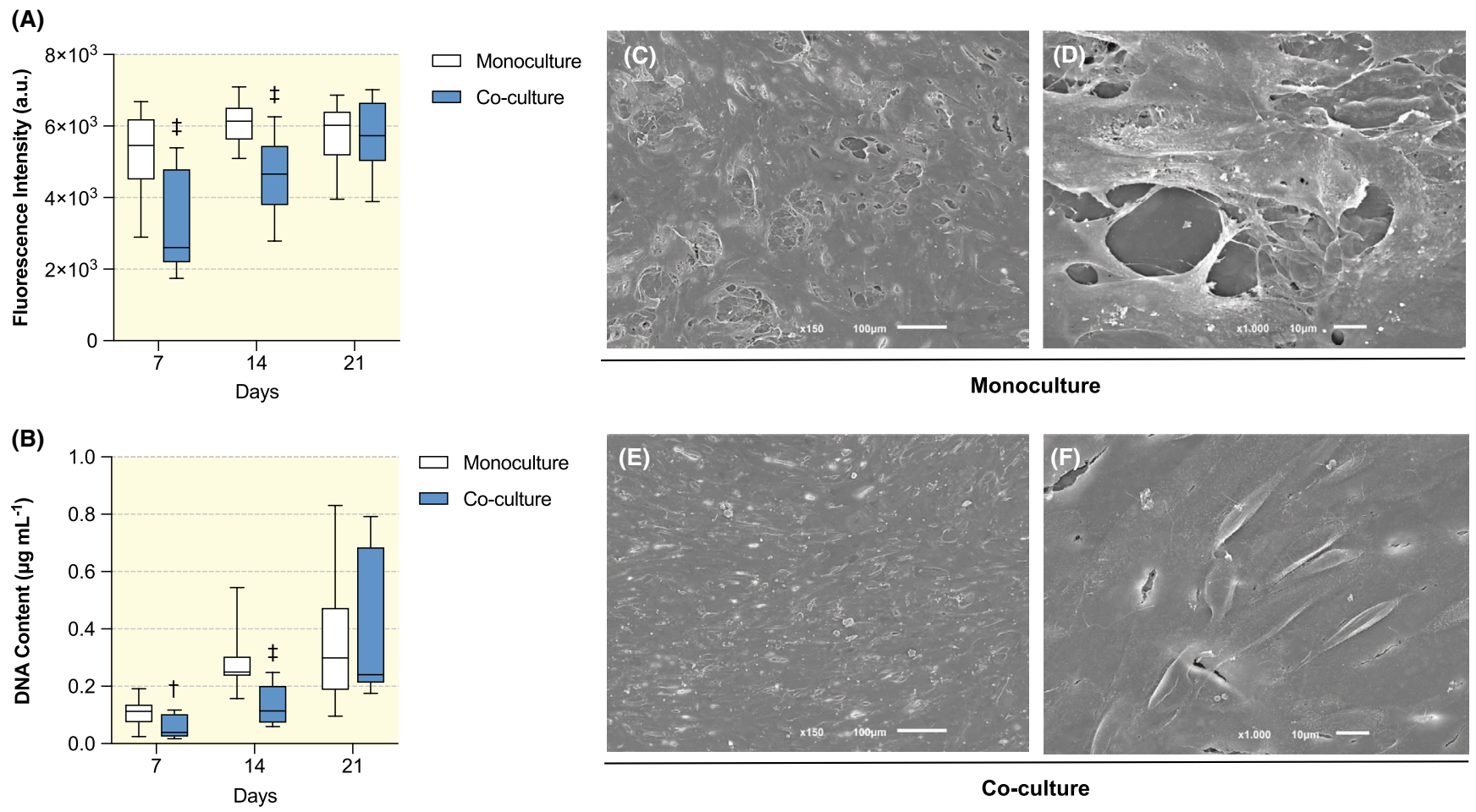

F I G URE 2 Behaviour of hRPC in contact with decellularized matrix membrane over 21 days. (A) Metabolic activity assay demonstrated by alamarBlue fluorescence after a 4 hours incubation period. Monocultures are represented by white bars, whereas co-cultures are represented by blue bars. Monocultures present higher values at 7 and 14 days, whereas after 21 days mean values are equal on both; (B) proliferation assay demonstrated by quantification of dsDNA over time. The same tendency as in viability assay is observed. Monocultures reach a plateau phase after 14 days culture, whereas in co-cultures values are increasing over time; ${ }^{\dagger} P<.01,{ }^{\ddagger} P<.001$ vs monoculture; (C-F) SEM was performed on fixed hRPCs to evaluate morphology and distribution after 21 days on the decellularized matrix membranes. Monocultures present a non-organized monolayer of cells, while in co-cultures hRPCs have organized themselves in a polarized manner, resembling natural epithelial organization and morphology. Scale bars: (C and E): $100 \mu \mathrm{m}$; (D and F): $10 \mu \mathrm{m}$

no statistical difference at 21 days of culture between monoand co-cultures for both assays. Indeed, results revealed that at 7 and 14 days, mean values and correspondent interquartile ranges were lower for co-cultures when comparing with monocultures, being this difference statistically relevant for DNA content measurements. These cells were already demonstrated to possess high clonogenic potential and self-renewal ability. In this work we demonstrate that this capacity is not influenced by the presence of the endothelial cells on the system. Indeed, the lower values observed in the first time points for co-cultures can be explained by the higher number of cells in the system for the same quantity of media. This allows higher proliferation in monocultures at early time points, reaching cell confluency faster. As a result, when comparing to co-cultures, a plateau phase is observed specially from day 14 to 21 for both assays in monocultures.

It was also important to evaluate how cells organize in this system. We previously observed the formation of a monolayer of epithelial cells on these membranes. ${ }^{16}$ However, this work was a proof of concept performed with a cell line, having its limitations when mimicking an in vivo mechanism. Herein, we intended to assess if the hRPCs followed the same tendency as previously observed in monolayer cultures.
Therefore, SEM was performed after 21 days on mono(Figure 2C,D) and co-cultures (Figure 2E,F). Contradicting the above-mentioned quantification results, we notice a huge difference on how cells organize in these membranes. In co-cultures, a homogenous and organized layer of cells was observed, with all cells oriented and resembling a natural epithelial monolayer. On the other hand, for monocultures, some parts of the fibres which compose the membranes can be seen, proving that cells were not able to form a homogeneous monolayer on this condition. Additionally, no cellular polarization is observed, being the cells present in a spiral and round-shaped morphology. In some sites, cells seem to be creating multilayered structures, which corroborates the higher values obtained for DNA content. Overall, these results demonstrate that hRPCs may be presenting a different phenotype when co-cultured with HUVECs.

\section{3 | Phenotypic characterization of hRPCs over time}

To understand the response of progenitor cells to the created system, their phenotypic expression was characterized 
simultaneously by qPCR and IF stainings of relevant tubular markers. Gene expression was analysed for genes encoding three different epithelial transporters: $\mathrm{Na} / \mathrm{K} / \mathrm{Cl}$ co-transporter (SLC12A1), amino acid transport protein (SLC3A1) and $\mathrm{Na} / \mathrm{H}$ exchanger (SLC9A3). Not only the presence of transporters but also the recapitulation of physiological functions is important. For that the expression $\gamma$-glutamyltranspeptidase $(G G T 1)$ was also measured, being this enzyme responsible for the supply of an adequate amount of glutathione, essential to maintain normal kidneys function. ${ }^{7}$ As demonstrated (Figure 3A-D), all the genes are upregulated at day 21 for mono- and co-cultures. We observe that the higher turnover happens essentially from day 14 to day 21 and that all of the genes follow the same tendency over time in monoand co-cultures. Indeed, between the two conditions, the only statistical difference happens in GGT1 expression, which is probably caused by variability between samples, since it is a unit-fold difference between the two conditions. The higher fold expression happens on $\mathrm{Na} / \mathrm{K} / \mathrm{Cl}$ co-transporter (with a mean of $\sim 30$-fold and $\sim 21$-fold expression for mono- and co-cultures, respectively) and on $\mathrm{Na} / \mathrm{H}$ exchanger (with a mean of $\sim 13$-fold and $\sim 15$-fold expression for mono- and cocultures, respectively). No differences in GAPDH expression were found with culture time and co-culture systems, validating our qPCR assay (Figure S1B).

Afterwards, we used fluorescence microscopy to directly confirm the presence of tubular-related proteins in co-cultures
(Figure 3E-H) and in monocultures (Figure S1C-F). We could observe that the proximal tubular transporters aquaporin 1 (AQP-1) and sodium glucose transporter (SGLT2) have a more homogeneous fluorescent staining (Figure 3E,F) when comparing to the distal convoluted tubule $\mathrm{Na} / \mathrm{Cl}$ co-transporter (NCC) and the thick ascending limb chloride channel Ka (CLC-NKA) (Figure 3G,h). Furthermore, by comparing with the immunofluorescent staining of the same transporters in monocultures (Figure S1C-F), a much more diffuse staining is shown in all transporters, with several cells evidencing no signal. Indeed, no green staining is shown for CLC-NKA. We experienced difficulties in obtaining clear confocal images caused by the autofluorescence of the ECM that strongly absorbs DAPI stain. ${ }^{16}$ Because of that, and since each membrane is different on its irregularity, we selected a representative site of the membrane in which we could obtain micrographs with sharper contrast. Protein content on the cell cultures was also measured over the 21 days of culture (Figure S1A). No statistical differences were found between mono- and co-cultures. However, an increase on protein content from day 7 to day 14 was observed for monocultures, which decreased after 21 days. Indeed, the same tendency was observed for the proliferation assay, and a plateau phase is observed from day 14 to 21 in monocultures. In contrast, proliferation in co-cultures was stable from day 14 to 21 and protein content values increased, a possible indicator of cellular differentiation.
(A)
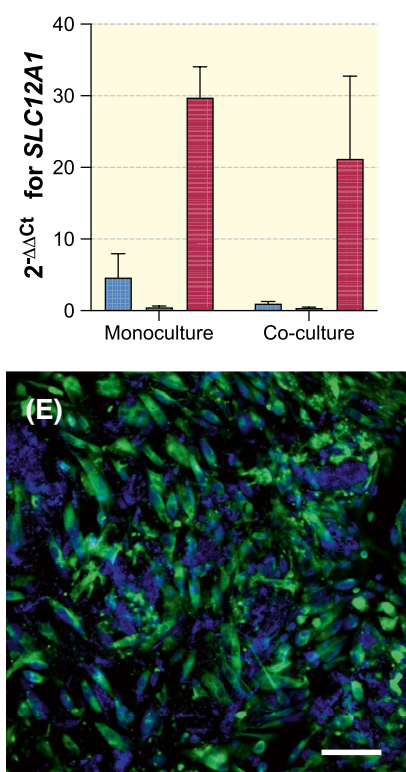

(B)
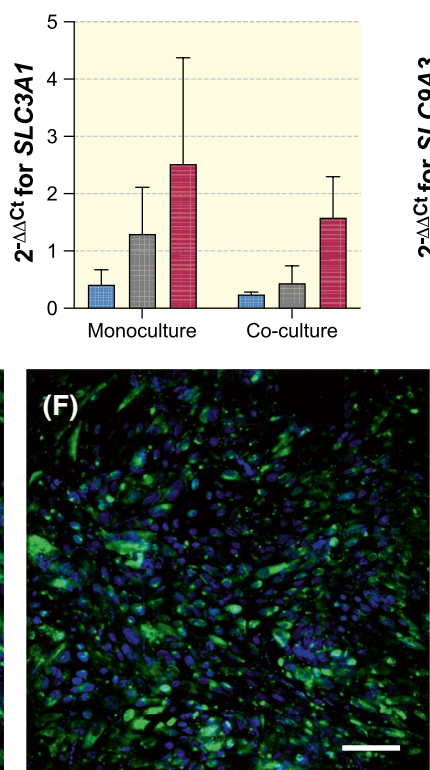

(C)
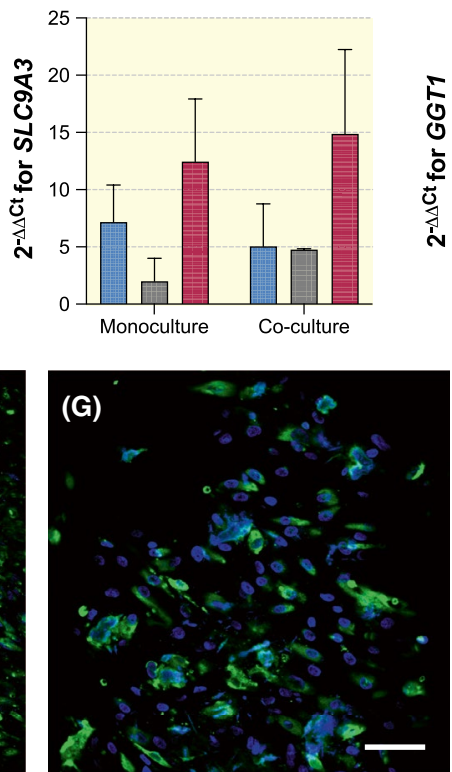

(D)
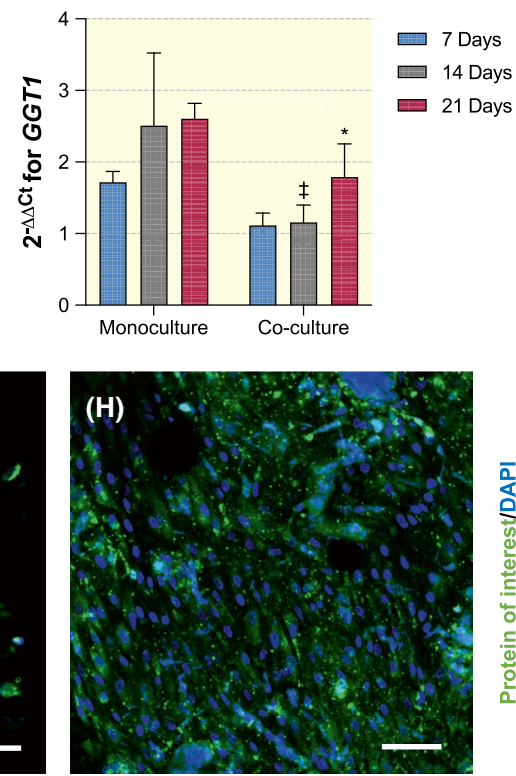

F I G U RE 3 Phenotypic characterization of hRPCs cultured on decellularized membranes. Gene expression of tubular-related markers: (A) SCL12A1 (Na/K/Cl co-transporter), (B) SLC3A1 (amino acid transport protein), (C) SLC9A3 (Na/H exchanger) and (D) GGT1 ( $\gamma$-glutamyltranspeptidase); ${ }^{*} P<.05,{ }^{\ddagger} P<.001$ vs monoculture; Tubular-related markers protein assessment of (E) AQP-1 (aquaporin-1), (F) SGLT2 (sodium glucose transporter 2), (G) NCC (Na/Cl co-transporter) and (h) CLC-NKA (chloride channel Ka) marked in green. Nuclei were stained with DAPI (blue). Scale bar: $100 \mu \mathrm{m}$. Both assays were performed simultaneously. All genes are upregulated at 21 days of culture with respect to their expression at day 0. Additionally, AQP-1 and SGLT2 had the most homogenous staining. These results suggest preferable differentiation of hRPCs on proximal tubular cells when in contact with the decellularized membranes 


\subsection{Polarization and maturity assessment on proximal tubule cells}

The ability of the RPTECs/HUVECs bilayer model to recapitulate renal function is highly dependent on the polarity and maturity of the seeded cells. For that, we have used imaging methods to analyse cellular organization and specific protein content after 7 days of bilayer seeding, corresponding to the 30th day of hRPCs differentiation stimulus. The previously detected phenomena for the hRPCs were also observed for hRPTECs, although the position of the endothelial cells had changed as well as the degree of differentiation of the hRPCs. As demonstrated in Figure 4A,B, epithelial morphology and distribution was highly influenced by the presence of the HUVECs, when comparing to monocultures (Figure S2A,B). The endothelial cells enhanced the formation of a polarized monolayer of epithelial cells, typical of renal morphology. ${ }^{14}$ SEM micrographs of higher magnification also revealed the formation of tight junctions connecting the neighbouring cells (signalled by circles) and short microvilli at the apical surface (signalled by arrows), although much more present in co-cultures (Figure 4B), confirming epithelial cell polarization in this system. Indeed, we calculated the percentage of cells expressing microvilli on mono- and co-cultures at 150x magnification SEM micrographs by ImageJ software (Figure 4D). The obtained values corroborate the above results, where $\sim 83 \%$ of the co-cultured cells were found to present microvilli on their cell surface, contrasting with a value of $\sim 21 \%$ for monocultured hRPTECs.

After observing a positive effect of the presence of HUVECs on hRPTECs monolayer formation, we evaluated if in co-cultures we could observe signals of enhanced maturity by IF staining of tubular-related proteins. Similar to what was observed in hRPCs culture, we could notice higher fluorescence intensity signal and an overall more organized cellular distribution on co-cultures (Figure 4D-G) than for monocultures (Figure S2C-F), which confirms the maturity observed in SEM micrographs. Also, fluorescence intensity differed for the different tubular segments, being much higher for proximal tubular markers in both conditions (AQP1 and SGLT2). However, in contrast to hRPCs culture, we could observe better fluorescence signal for NCC and CLC-NKA markers, indicative of simultaneous differentiation on epithelial cells of the distal tubule and the thick ascending limb
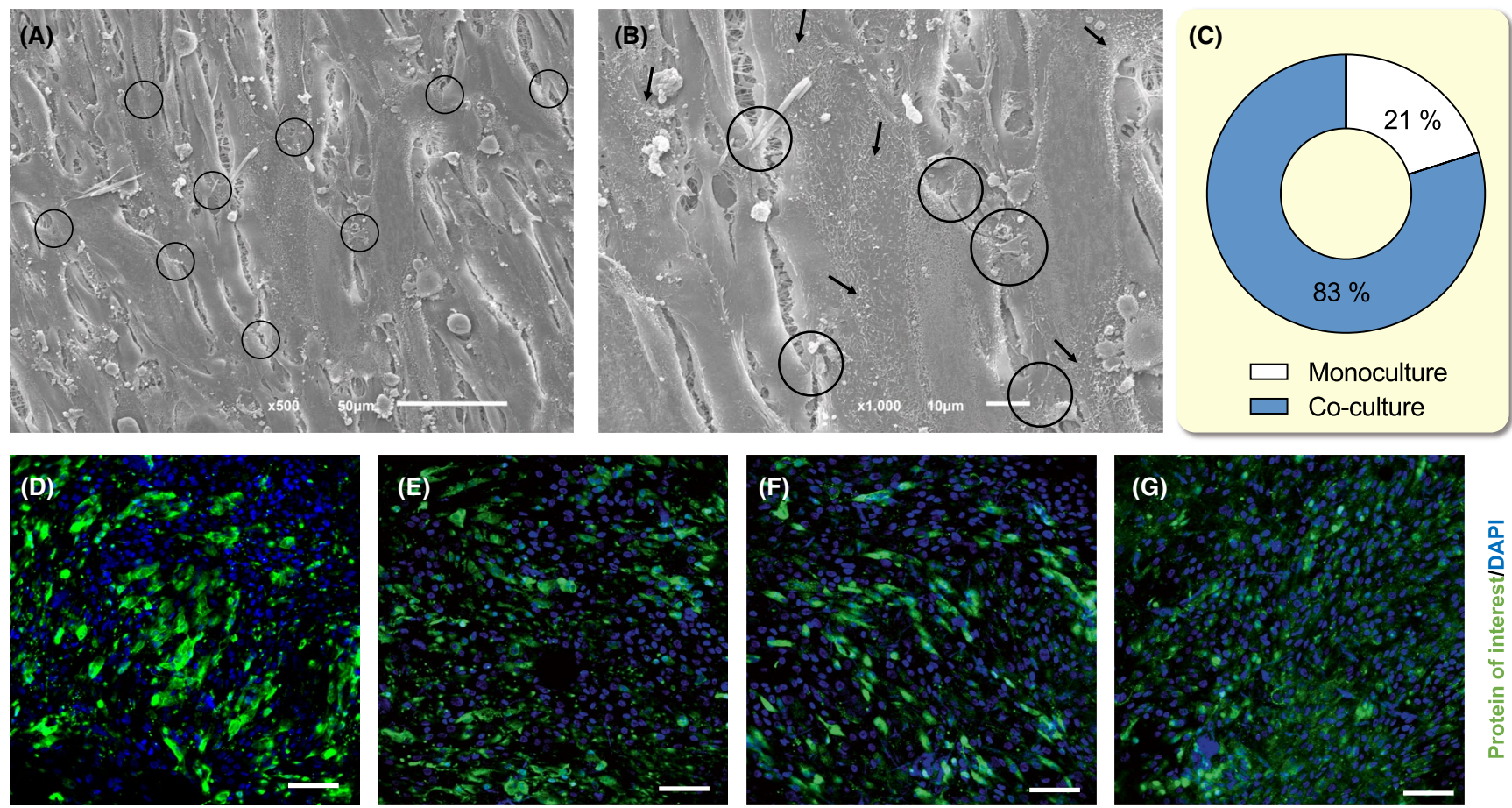

F I G U RE 4 Assessment of hRPTECs maturity and polarization after 7 days of bilayer assembling. (A-B) SEM micrographs of hRPTECs co-cultured with HUVECs. These cells organized in a homogenous-oriented monolayer. Tight junctions characteristic of fully maturated epithelial tubular cells can be observed (indicated by circles) as well as microvilli (indicated by arrows). These micrographs suggest cellular polarization with the apical surface towards the top compartment, accurately mimicking tubular organization; (C) Percentage of cells expressing microvilli on mono(white) and co-cultures (blue). Values were obtained by cell counting on ImageJ software; (D-G) Tubular-related markers protein assessment of (D) AQP1 (aquaporin 1), (E) SGLT2 (sodium glucose transporter 2), (F) NCC (Na/Cl co-transporter) and (G) CLC-NKA (chloride channel Ka) marked in green. Nuclei were stained with DAPI (blue). Results demonstrate the presence of proteins corresponding to several nephron segments on hRPTECs, indicating successful maintenance of fully differentiated epithelial cells on our membranes. Scale bars: (A) $50 \mu \mathrm{m}$; (B) $10 \mu \mathrm{m}$; (D-G) $100 \mu \mathrm{m}$ 
of the loop of Henle. Overall these results suggest a good differentiation onto the renal tubular lineage on our co-culture system, with the presence of specialized cells of different segments of the tubule.

\subsection{Characterization of proximal tubule physiological functions}

To understand and characterize the developed system, we investigated Human Serum Albumin (HSA) and inulin transport (Figure 5A,B, respectively). These compounds were loaded into the upper compartment and their kinetics of diffusion were measured over time. We noticed that after 5 hours, HSA uptake was more pronounced in co-cultures versus monocultures ( $60 \%$ vs $\sim 40 \%$ uptake). Inulin leakage was measured as an indicator of the barrier function. We noticed a slight increase in the leakage during the experiment both in mono- and co-cultures, which was more pronounced in monocultures ( $\sim 12 \%$ vs $\sim 8 \%$ in co-cultures). However, this increase was expectable because of the over concentrated solution of inulin introduced in the system and the necessary manipulation of the system between the time points. It can also be observed that in all time points leakage values for monocultures are higher than in co-cultures, being statistically different at the 5 hours time point. Comparing these results with the values obtained for non-seeded membranes, we noticed that there was no uptake of HSA, being the values around $0 \%$ or negative, which could be explained by medium evaporation for the same amount of probe throughout the time points. Inulin leakage after 5 hours was $\sim 50 \%$ for membranes with no cells (data not shown), which indicates that the membranes alone are also selectively permeable. The results obtained for cell-seeded membranes indicate selective reabsorption characteristics of our bilayer model.

\subsection{Modelling of drug-induced nephrotoxicity}

A major goal in developing in vitro models is to have the recapitulation of physiological functions and disease phenotypes to better predict drug toxicity in humans. We therefore administered the known chemotherapeutic drug and nephrotoxin cisplatin in the top compartment of our co-culture system in different dosages. We evaluated cellular viability by live/dead assay in co-cultures (Figure 6A-D) and monocultures (Figure S3A-D) and by MTS (Figure 6I), comparing to a control condition $(0 \mu \mathrm{M}$ of cisplatin). Phalloidin and DAPI staining was also performed on fixed mono- (Figure S3E-H) and co-cultures (Figure 6E-H). We could observe that, with the increasing concentration of cisplatin, cellular viability was reduced in $\sim 80 \%$ for monocultures and $\sim 90 \%$ for co-cultures when the higher concentration of the drug was administered. Although no significative differences were observed between both conditions, we could notice that co-cultured cells reacted quicker to the drug than in monocultures, which can be as a result of the higher abundance of active transporters mediating drug uptake. Live/dead micrographs indicate the abundance of dead cells by the internalization of propidium iodide. These micrographs corroborate the above MTS results, where a higher red and lower green fluorescence staining can be detected with the increasing concentration of cisplatin, comparing with the control condition (Figure 6A). Additionally, on monocultures (Figure S3), some parts of the cellular body can still be distinguished even after the administration of a higher cisplatin dose,
(A)

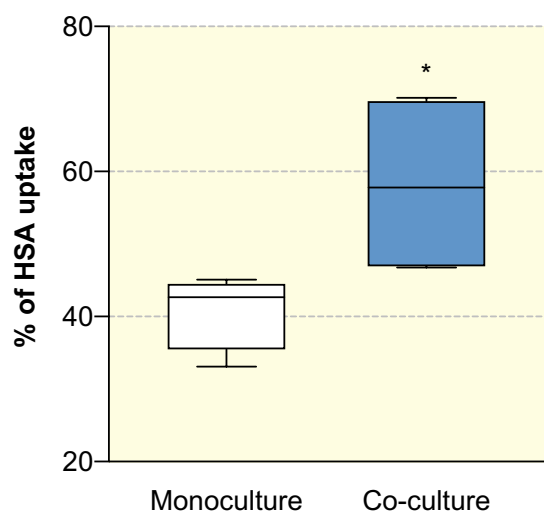

(B)

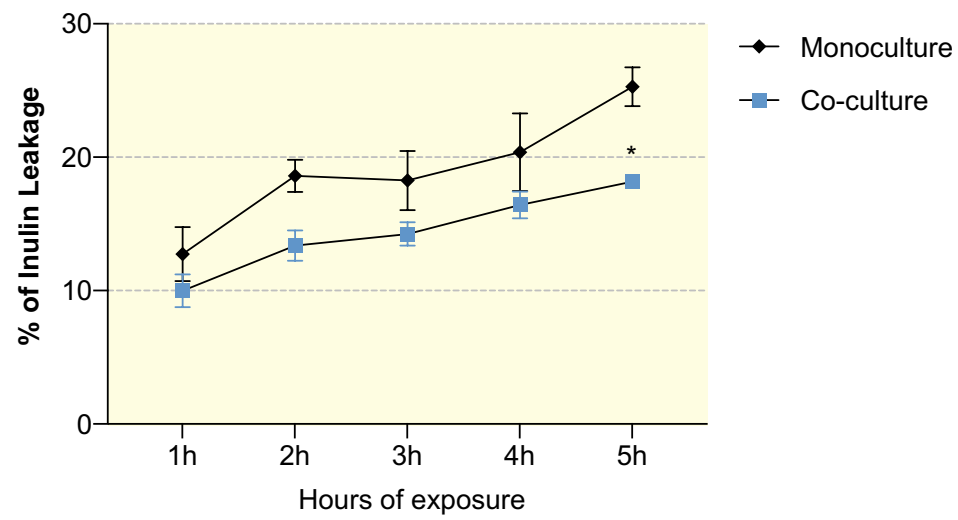

F I G U R E 5 Functional assays on the bilayer model. (A) Human serum albumin (HSA) uptake of hRPTECs cultured on mono- or co-cultures. A solution of FITC-conjugated HSA with a known concentration was introduced on the top compartment. After 5 h, the concentration of the remaining compound was extrapolated from its fluorescence intensity; (B) Percentage of inulin leakage obtained for mono- and co-cultures. FITCconjugated inulin at a known concentration was introduced on the top compartment. Throughout the $5 \mathrm{~h}$ time points, the fluorescence intensity of the compound present on the bottom compartment was measured; $* P<.05$. These results are indicative of higher endocytic activity as well as higher resistance to compound leakage on epithelial cells cultured in the bilayer model, rather than in monocultures 
$0 \mu \mathrm{m}$
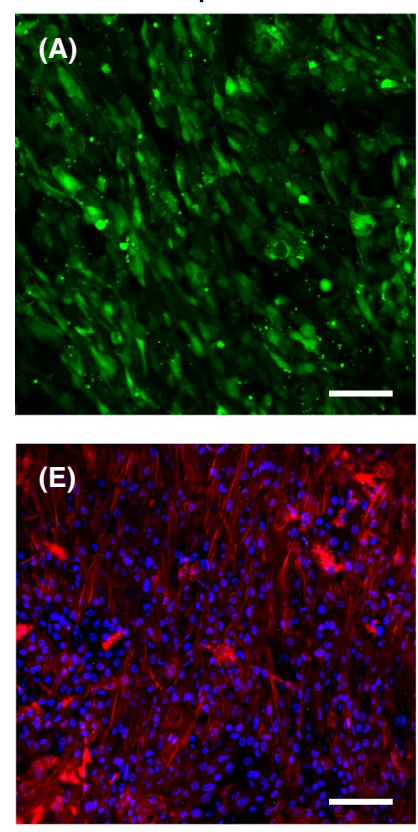

$50 \mu \mathrm{m}$
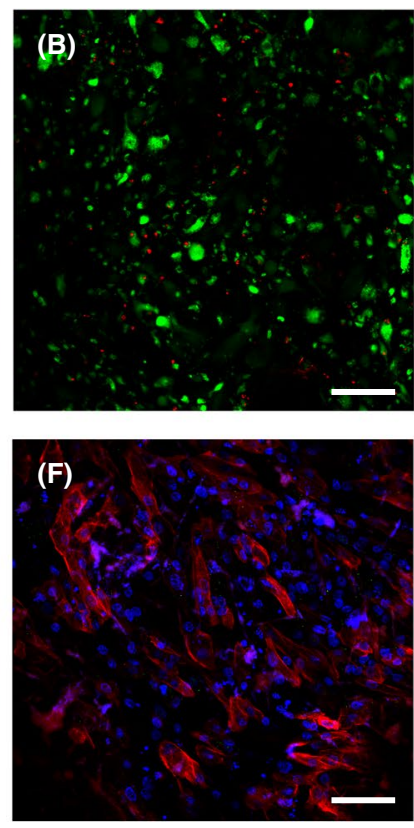
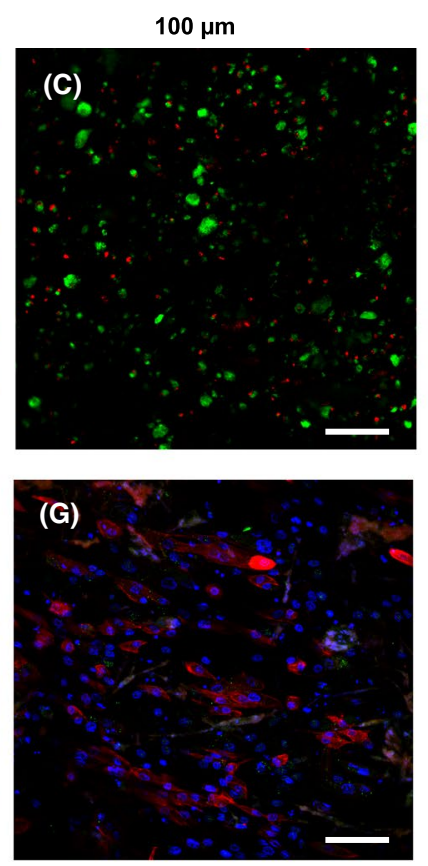

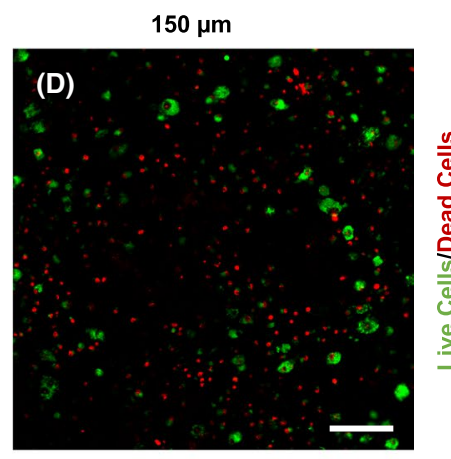

(H)

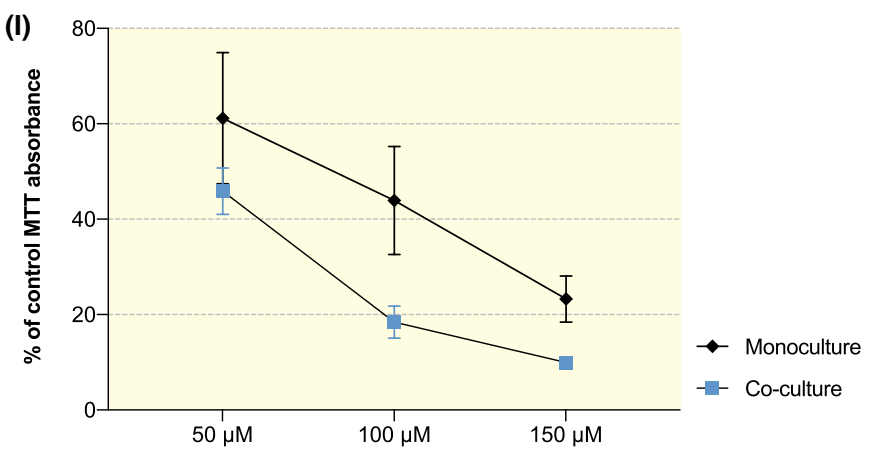

F I G U RE 6 Cisplatin-induced nephrotoxicity model. Cisplatin was dissolved in cell culture medium in different concentrations and introduced in the cell culture system to assess cellular damage. (A-H) Qualitative results for viability on co-cultures were obtained by immunofluorescence micrographs of live/dead assay and Phalloidin/DAPI staining of fixed cells. Cells were administered with $0 \mu \mathrm{M}(\mathrm{A}, \mathrm{E})$, $50 \mu \mathrm{M}(\mathrm{B}, \mathrm{F}), 100 \mu \mathrm{M}(\mathrm{C}, \mathrm{G})$ and $150 \mu \mathrm{M}(\mathrm{D}, \mathrm{H})$ of cisplatin. Live/Dead assay was performed by incubating cells with Calcein AM (stains green for live cells) and PI (stains red for dead cells). DAPI/Phalloidin staining was performed after cell fixation in formalin. Cytoskeleton is stained by phalloidin (red) and nuclei are stained by DAPI (blue). (i) Quantitative results for viability were obtained by MTS assay. No significative differences were found between mono- and co-cultures. These assays revealed that hRPTECs cultured on the bilayer model were more sensitive to the presence of cisplatin, which uptake occurs in proximal tubules. Scale bars: $100 \mu \mathrm{m}$

which does not happen in co-cultures, where scarce green staining appears. This is again indicative of a higher epithelial differentiation degree. However, the demonstrated green staining is often accompanied by red staining in the same cell, suggesting that cells may be entering apoptosis. Phalloidin and DAPI staining on fixed mono- and co-cultures corroborates the above results, where lower red staining for phalloidin is observed with the increasing concentration of cisplatin.

\section{3 | DISCUSSION}

In this work, we focused in developing and characterizing a physiologically relevant in vitro tubular bilayer model incorporating both endothelial and epithelial cells. By using an electrospun membrane, we intended to mimic orientation, separation, porosity and composition of the basement membrane found in vivo (Figure 1). The large surface area obtained by electrospinning processing has already demonstrated to allow stronger cell adhesion and proliferation, ${ }^{22,23}$ which was our main goal when fabricating these membranes. They were previously studied by our group, where we demonstrate functionality of the proteins included on the membrane, by comparing cellular behaviour on membranes which had no dKECM on their composition. ${ }^{16}$ Herein, we intended to further explore their potential by using co-cultures of endothelial and renal progenitor cells (the precursors of tubular epithelial cells). The obtained results for quantitative cellular 
assays reveal that hRPCs do not demonstrate any kind of preference for being in co-culture or monoculture (Figure 2), which can be attributed to the lack of evidence showing any beneficial effect on having endothelial cells boosting the performance of renal progenitor cells. However, we demonstrated that the formation of monolayer was remarkably enhanced by adding endothelial cells to the system. The assessment of ultrastructure by SEM revealed hallmarks of an epithelial-like morphology, showing the importance of the co-cultures in this kind of studies. We also characterized the phenotypic expression by qPCR and IF staining methods. All genes were upregulated after 21 days, revealing hRPCs differentiation into tubular epithelial cells (Figure 3). The major fold increased expression was observed for SLC12A1 and $S L C 9 A 3$, which encode $\mathrm{Na} / \mathrm{K} / \mathrm{Cl}$ co-transporter and the $\mathrm{Na} / \mathrm{H}$ exchanger respectively. Sodium transport in the nephron is mainly driven by these two transporters, which explains the higher expression of these genes. The upregulation of $S L C 3 A 1$ and GGTI was also demonstrated, which is indicative of a higher maturation of these cells, since both genes encode proteins responsible for amino acid transport on the tubular epithelial brush border. IF revealed consistent staining of proximal tubular markers AQP-1 and SGLT2, whereas the signal for NCC and CLC-NKA, markers for the distal tubule and the thick ascending limb, was lower. Furthermore, by comparing these results to that of the monocultures, we observe overall lower IF signal of all markers (Figure S1). These findings demonstrate not only a preference for hRPCs differentiation into proximal tubular cells but also increased phenotypic markers provoked by the presence of endothelial cells. Moreover, the cells were able to express tubular-related genes and proteins at day 21 of culture, contrasting with a previously published work, in which a 30-day culture was necessary for fully tubular differentiation of hRPCs. ${ }^{17}$

To further evaluate how hRPCs potentiate the use of the electrospun membrane as a model of a natural renal filtration barrier, we have also studied co-cultures of hRPTECs with HUVECs, by growth factor-induced differentiation of hRPCs. Figure 4 clearly demonstrates the ultrastructure, polarization and the presence of characteristic transporters of the renal epithelial cell lineage. ${ }^{24}$ Brush-border microvilli and tight junctions are abundant in all of the cells co-cultured on the membranes, which are polarized according to the organization of the in vivo tubule. We can observe the apical membrane turned to the top compartment which suggests the basolateral membrane to be in contact with the decellularized kidney ECM, similar to what happens with in vivo BMs. By IF, markers correspondent to different sections of the tubule were assessed to understand the heterogeneity of the population. Less fluorescence intensity was found on CLC-NKA, which is explained by the basolateral location. However, all of the markers were found present on our membranes, showing differentiation towards other epithelial phenotypes rather than proximal tubule cells, which were not previously demonstrated with renal progenitor cells. Higher fluorescence intensity is always found with the presence of HUVECs on the system, comparing with monocultures (Figure S2), demonstrating once more the value of the epithelial cells on modulating cellular phenotype.

The adopted polarized morphology of hRPTECs when in co-culture with HUVECs allowed the study of renal-specific transport functions across the bilayer model, similar to the complete pathway which water and solutes cross when reabsorbed from the tubular lumen to the blood flow. We have demonstrated consistent values for HSA uptake and inulin leakage (Figure 5). Indeed, we were able to obtain lower values for inulin leakage than what is reported in the literature for transwell systems. ${ }^{10}$ We were also able to obtain higher values for HSA uptake comparing with tubule models including immortalized hRPTECs instead of primary lineages. ${ }^{25}$

One of the major goals on developing a reliable in vitro model of the tubule is to have a consistent response to drugs which are known to induce nephrotoxicity. In this way, it would be possible to predict drug toxicity on humans. Cisplatin is a known nephrotoxic drug that accumulates in the renal parenchyma by active transport into proximal tubular cells. ${ }^{26,27}$ Herein, we have demonstrated that hRPTECs exhibit a dose-dependent toxic response to cisplatin with higher fidelity when cultured on the bilayer model (Figure 6). Indeed, by live/dead assay micrographs we demonstrate that cisplatin induced less cellular damage in monocultures which was also corroborated by phalloidin cytoskeleton staining. These results suggest that our model should provide specific and reliable measurements of drug toxicities in vitro.

The main aim of an epithelial-endothelial bilayer system is having the formation of single-layered epithelia and their maintenance for long periods of time. We were able not only to develop a system capable of recapitulating morphology and physiological functions but also a system which is capable of maintaining the maturity of differentiated cells. Other authors developed different systems in order to mimic ECM compositions and spatial organization of the tubular basement membrane, including bioprintring, ${ }^{25,28,29}$ microphysiological systems ${ }^{7}$ and tubule-on-a-chip technology. ${ }^{30}$ Herein, we employ a different strategy, where a similar composition to the human kidney ECM is used to give a proper stimulus to the cultured cells. The importance of the ECM on the maintenance of these monolayers was already described. ${ }^{5}$ Indeed, changes in the ECM in the tubule and the surrounding interstitium are associated with pathological conditions, including inflammatory processes and renal fibrosis. Our system enabled obtaining the in vivo variability by the incorporation of natural kidney ECM on electrospun membranes. Besides, no additional treatment was necessary to mimic porosity, since that electrospinning enables obtaining porous membranes, suitable for solute and water passage between the two layers. ${ }^{31}$ 
However, we do think that there are further developments to be performed, including employing specific renal endothelial cells, which could have recapitulated in a more relevant manner the organization and phenotype of an interface between tubular and vascular poles. Indeed, in the future, we intend to not only have a mature renal cellular phenotype on the bilayer but also introduce flow-induced shear stress to the model, to better understand and mimic physiological conditions. We also will work on understanding what is the exact stimulus that the proteins present on our membrane are giving to the cells and which cellular pathways are being activated. Nevertheless, we do think that this biomimetic system constitutes a powerful tool not only for drug toxicity screening but also for increasing our knowledge on molecular mechanisms that underlie kidney function and disease. To the best of our knowledge, this is the first demonstration of a co-culture system on decellularized kidney membranes and also the first time that hRPC-derived epithelial cells were co-cultured and maintained in a differentiated state in vitro.

\section{4 | MATERIALS AND METHODS}

\subsection{Experimental Design}

Kidneys were decellularized and the matrix was further processed by milling and freeze-drying. Membranes of 1:1 decellularized kidney ECM (dKECM) to poly (caprolactone) (PCL) were obtained by electrospinning, after solubilizing both materials in HFIP. A brief scheme of the processing steps is represented in Scheme 1.
For the cell culture assays, human renal progenitor cells (hRPCs) were used to validate the model. We applied two separate and yet complementary approaches; initially, hRPCs were assessed for their spontaneous differentiation into cells of the tubular lineage. We compared how these cells behaved when in mono- or co-culture with human umbilical vein endothelial cells (HUVECs, Scheme 1A). In a parallel study, the same cells were stimulated to differentiate in 2D substrates into renal proximal tubular epithelial cells (hRPTECs) and were again co-cultured, each cell type in one side of the membrane, creating a bilayer model of an in vivo tubule (Scheme 1B).

\section{2 | Processing of decellularized kidney electrospun membranes}

Porcine kidneys were obtained from a local slaughterhouse. The decellularized matrix was processed as previously described, ${ }^{16}$ with minor modifications. Briefly, kidney portions were immersed in a solution of $1 \%$ SDS before being changed to another solution of $1 \%$ Triton X-100. The solutions were placed in an orbital shaker and changed twice a day. After the decellularization process, the matrix was washed, freeze-dried and reduced to powder. The powdered matrix was solubilized in HFIP $(1,1,1,3,3,3-$ Hexafluoro-2-propanol $\geq 99 \%$ ) (Sigma-Aldrich) at a concentration of $100 \mathrm{mg} / \mathrm{mL}$. Polycaprolactone (PCL, $80 \mathrm{kDa}$, Sigma Aldrich) was dissolved in the same reagent at a concentration of $15 \%(\mathrm{w} / \mathrm{v})$. Both solutions were mixed 1:1 and electrospinning process was performed as previously

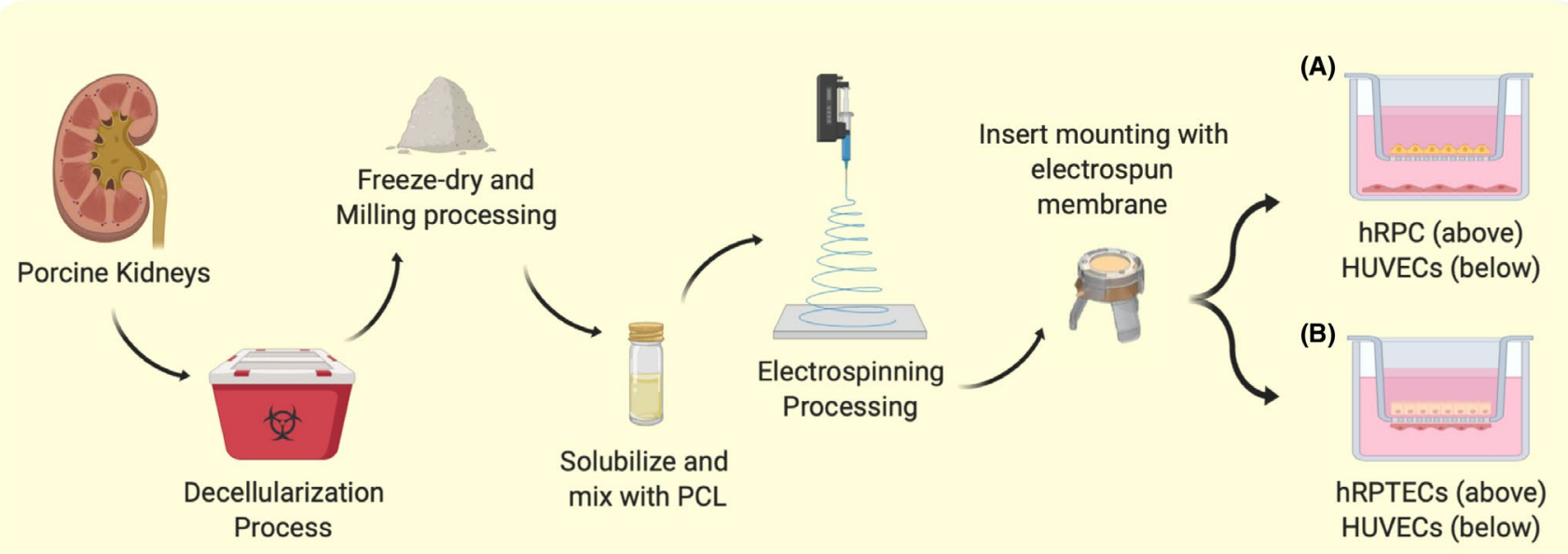

SCHE ME 1 Schematic representation of decellularization process and membranes preparation procedure. Porcine kidneys are obtained on a local butcher. They were chopped and decellularized in different detergent solutions before being processed by freeze drying and with a cryogenic mill to obtain a powder. In order to have a suitable solution for electrospinning process, the powdered matrix is blended with a PCL solution at 1:1 ratio in an organic solvent. After the production of the membranes, these are mounted in 24-well inserts and sterilized. In this work, we developed two kinds of cultures (A) to evaluate hRPCs interaction with HUVECs and (B) a bilayer model composed of differentiated epithelial tubular cells (hRPTECs) and HUVECs to better mimic the in vivo tubulointerstitial environment 
described. ${ }^{16}$ The membranes were cut in squares and placed on 24-well inserts (CellCrown) before being sterilized by immersion in $70 \%$ ethanol and 30 minutes exposure to UV on each insert side.

\section{3 | Biochemical and immunohistochemistry analysis of the dKECM}

Samples were fixed for 30 minutes with $10 \%$ neutral buffered formalin before being embedded in OCT compound (Bio-optica). After this, they were snap freezed in liquid nitrogen and cut into $10 \mu \mathrm{m}$ sections using a cryostat. Sections were incubated with primary antibodies for immunofluorescent analysis as follows: anti-Collagen IV (1:100; ab6586), anti-Fibronectin (1:100, ab2413) and anti-Collagen I (1:40, ab765). Secondary antibody Alexa Fluor 488 (1:500) was incubated with 4,6-diamidino-2-phenylindole (1:1000, DAPI) for 1 hours at RT before being analysed under a confocal microscope.

Total protein content was quantified with Pierce Coomassie (Bradford) Protein Assay Kit (Thermo Scientific), according to the manufacturer's instructions. After sample incubation with the substrate, absorbance was measured at $562 \mathrm{~nm}$ in a microplate reader. The concentration of total protein in the samples was extrapolated using a standard curve of bovine serum albumin.

\section{4 | DKECM electrospun membranes morphological and structural characterization}

The morphology of the membranes was observed by scanning electron microscope (SEM, JSM-6010 LV, JEOL). Samples were sputter coated with gold prior to analysis and micrographs were obtained using an accelerating voltage of $10 \mathrm{kV}$. The fiber average diameter was statistically calculated using a computed image analyser (ImageJ) by measuring at least 200 fibres randomly in 3 samples from different fabrication batches. Membranes cross sections were obtained by immersing fibres in liquid nitrogen and cutting immediately to avoid deformation. Average membrane thicknesses were calculated from SEM micrographs of cross sections with ImageJ software.

Membrane porosity was determined as previously described. ${ }^{32}$ A liquid displacement technique was used a porosity was calculated following Equation (1), where $V_{1}$ is the initial liquid volume, $V_{2}$ is the volume after soaking the membrane and all the pores are filled and $V_{3}$ is the volume after membrane soaking and removal.

$$
\text { Porosity }(\%)=\frac{V_{1}-V_{3}}{V_{2}-V_{3}} \times 100
$$

To determine weight loss and water uptake of the membranes, these were initially weighted $\left(m_{i}\right)$ and soaked in an isotonic solution of sodium chloride (Sigma Aldrich). After, membranes were incubated at $37^{\circ} \mathrm{C}$ for 30 days. These conditions were optimized to simulate the human body environment. After known periods of time, membranes were weighted in their wet state $\left(m_{w}\right)$ and the percentage of water uptake was calculated using the Equation (2). Membranes were then placed on the incubator for another hour to dry before being weighted again $\left(m_{f}\right)$. Weight loss percentage was calculated using Equation (3).

$$
\begin{gathered}
\text { Water uptake }(\%)=\frac{m_{w}-m_{i}}{m_{i}} \times 100 \\
\text { Weight loss }(\%)=\frac{m_{i}-m_{f}}{m_{i}} \times 100
\end{gathered}
$$

\section{5 | Isolation and characterization of human renal progenitor cells (hRPC)}

Human kidney fragments were obtained in agreement with the Ethical Committee on human experimentation of the Azienda Ospedaliero-Universitaria Careggi, Florence, Italy. All subjects gave their informed consent for inclusion before they participated in the study. The study was conducted in accordance with the Declaration of Helsinki, and the protocol was approved by the Ethics Committee on Human Experimentation of the Azienda OspedalieroUniversitaria Careggi, Florence, Italy (project identification code 2015/0009082 from 25/03/2015). Human RPCs were obtained and cultured as previously described with minor modifications. ${ }^{17}$ Briefly, the cortex was minced before proceeding to an enzymatic digestion with collagenase solution type IV $750 \mathrm{U} / \mathrm{mL}$ (Sigma Aldrich) for 45 minutes at $37^{\circ} \mathrm{C}$. After digestion, the solution was sieved through graded mesh screens (60 and 80 mesh). The cellular suspension was collected, washed and plated on petri dish containing complete medium. The medium is composed by Microvascular Endothelial Cell Growth Medium (EGM-MV, Lonza) supplemented with 20\% HyClone Fetal Bovine Serum (FBS, GE Healthcare). After 4-5 days of culture, cells that adhered to the plate were subcultured. Cultures were checked for simultaneous expression of CD133 and CD24 by flow cytometry at different passages, according to previously published methods for characterizing hRPCs. ${ }^{17}$

\section{6 | Renal endothelium-epithelium mono and bilayer seeding}

HRPCs were seeded on the membranes at a density of $3 \times 10^{3}$ cells/insert in endothelial basal medium (EBM, Lonza) 
supplemented with 10\% FBS (HyClone, GE Healthcare) and expanded for 21 days. For co-culture studies, primary HUVECs (Cascade Biologics, Inc) were plated at a density of $7.5 \times 10^{3}$ cells/well in the day before hRPCs were seeded. The cell seeding concentration was optimized to obtain a confluent monoculture of hRPCs at the end of the culture time.

For cell culture studies with hRPTECs, differentiation on tissue culture plastic was performed before cell seeding on the inserts. Briefly, hRPCs were cultured in Renal Epithelial Cell Growth Medium (REGM, Lonza) supplemented with hepatocyte growth factor (HGF, Peprotech) at a concentration of $50 \mathrm{ng} / \mathrm{mL}$. HUVECs were seeded on inverted inserts at a density of $2 \times 10^{5}$ cells $/ \mathrm{mL}$ and allowed to attach 3 hours before being reverted. According to previously published reports, hRPCs take 30 days to differentiate into hRPTECs, when in specific cell culture conditions. ${ }^{19}$ Therefore, hRPCs with 23 days of differentiation supplementation were cultured at a density of $1.3 \times 10^{5}$ cells $/ \mathrm{mL}$ on the reverse side of the HUVECs insert for 7 days. The medium consisted of a mixture of HGF-supplemented REGM with EBM plus $10 \%$ of FBS.

\section{7 | Evaluation of cellular viability, proliferation and morphology}

At 7, 14 and 21 days of culture, the viability of hRPCs was assessed using alamarBlue (BioRad) assay. Membranes with seeded hRPCs were removed from the inserts, washed with DPBS and placed on new 24 well-plates with fresh cell medium containing $10 \%$ of alamarBlue reagent. After an incubation period of 4 hours at $37^{\circ} \mathrm{C}$, the supernatant was used to determine the fluorescence intensity in a microplate reader (excitation: 530/25 nm; emission: 590/25 nm). To evaluate hRPCs proliferation over time, dsDNA quantification was performed. Briefly, after the viability assay, constructs were rinsed in DPBS to release the alamarBlue dye. After, specimens were immersed in Milli-Q water and cells were lysed by thermal and osmotic shock. The resulting supernatant was used for the quantification with Picogreen dsDNA Kit. The fluorescence of each specimen was measured using a microplate reader (excitation: $485 \mathrm{~nm}$; emission: $528 \mathrm{~nm}$ ). The DNA concentration for each sample was calculated using a standard curve. The same supernatants were used to perform protein content measurements with Micro BCA protein assay kit (ThermoFisher) according to the manufacturer's instructions. After 2 hours of sample incubation with the substrate, absorbance was measured at $562 \mathrm{~nm}$ in a microplate reader. The concentration of total protein in the samples was calculated using a standard curve of Bovine Serum Albumin.

To analyse cellular morphology and the formation of a monolayer, after 21 days of culture, membranes were washed with DPBS and fixed in $2.5 \%$ glutaraldehyde (Sigma Aldrich) for 1 hour at $4{ }^{\circ} \mathrm{C}$. After the fixation step, they were washed again and dehydrated through the exposure to a gradient of alcohol concentrations. Samples were sputter coated with gold prior to analysis and micrographs were obtained using a scanning electron microscope (SEM, JSM-6010 LV, JEOL).

\section{8 | Immunofluorescence of renal epithelial-related proteins}

Both hRPCs and hRPTECs were fluorescently labelled with antibodies directed against markers for differentiated epithelia and transport proteins. Briefly, cell-loaded membranes were fixed with $10 \%$ neutral buffered formalin for $1 \mathrm{~h}$ at $4{ }^{\circ} \mathrm{C}$ and then washed in PBS. The blocking step was performed for 30 minutes incubating the membranes in $3 \%$ bovine serum albumin (BSA, Sigma Aldrich) solution. Primary antibodies were diluted in blocking solution and incubated overnight at $4^{\circ} \mathrm{C}$. Epithelial protein analysis was evaluated with antibodies against the sodium-dependent glucose transporter (SGLT2, abcam, 1:30), aquaporin-1 (AQP-1, abcam, 1:100), chloride channel Ka (CLC-NKA, Santa Cruz Biotechnology, 1:25) and $\mathrm{Na} / \mathrm{Cl}$ co-transporter (NCC, Santa Cruz Biotechnology, 1:25). Samples were washed in PBS and incubated with fluorescent labelled secondary antibodies Alexa Fluor 488 against the specific primary antibody species at 1:500 concentration. DAPI (Sigma Aldrich, 1:1000) was incubated along with the secondary antibody. After several rinses in PBS, constructs were mounted with VECTASHIELD (Vector Laboratories) and analysed under Leica SP5 AOBS confocal microscope (Leica, Wetzlar, Germany) equipped with a Chameleon Ultra-II two-photon laser (Coherent, Milan, Italy). Images were recorded digitally and further processed using LASX software (Leica).

\section{9 | Real-time quantitative polymerase chain reaction (RT-PCR) of tubular- related genes}

Total RNA was extracted using RNeasy Microkit (Qiagen) and quantified using a Nanodrop spectrophotometer (ThermoFisher Scientific). Briefly, after 7, 14 and 21 days, membranes with attached hRPCs in monoculture or in coculture systems were removed from the inserts and immersed in buffer RTL (acquired from the kit) containing $\beta$-mercaptoethanol for efficient cell lysis. After extraction, 52 ng of RNA was retrotranscribed using TaqMan Reverse Transcription Reagents (ThermoFisher) following manufacturer's instructions, in a final volume of $17 \mu \mathrm{L}$. TaqMan RT-PCR was performed as previously described using a 7900HT Real-Time PCR System (Applied Biosystems). ${ }^{33}$ $\mathrm{Na} / \mathrm{K} / \mathrm{Cl}$ co-transporter (SLC12Al), amino acid transport 
protein (SLC3A1), $\gamma$-glutamyltranspeptidase $(G G T 1)$ and $\mathrm{Na} / \mathrm{H}$ exchanger (SLC9A3) quantification was performed using commercially available Assay on Demand kits (Applied Biosystems). Fold expression (FE) was determined using the equation $F E=2^{-\Delta \Delta \mathrm{Ct}}$, where $\Delta \mathrm{Ct}=\mathrm{Ct}$ gene of interest $-\mathrm{Ct}$ housekeeping gene, glyceraldehyde-3-phosphate dehydrogenase $(\mathrm{GAPDH})$ and $\Delta \Delta \mathrm{Ct}=\mathrm{Ct}$ sample of interest $-\mathrm{Ct}$ control sample, which was considered the gene expression level at the seeding day.

\subsection{0 | Paracellular permeability and albumin reabsorption studies}

At day 7 of co-culture of hRPTECs and HUVECs, $400 \mu \mathrm{L}$ of media containing $25 \mu \mathrm{g} / \mathrm{mL}$ of fluorescein isothiocyanate (FITC)-conjugated inulin (Sigma Aldrich) was applied to the top compartment, while $600 \mu \mathrm{L}$ of medium was applied to the bottom compartment. Cells were incubated at $37^{\circ} \mathrm{C}$ for 5 hours. Every hour, samples were drawn from both compartments and fluorescence intensity was measured in a microplate reader (excitation: 485/25 nm; emission: 530/25 nm). For albumin uptake assay, FITC-conjugated human serum albumin (HSA) was applied to the top compartment at a concentration of $40 \mu \mathrm{g} / \mathrm{mL}$ and the initial fluorescence was measured in the total volume of the well. After 5 hours, inserts were drawn from the wells and the remaining concentration of HSA in the well was measured as above mentioned. The leakage and absorption studies were calculated as the percentage of the initial concentration.

\subsection{1 | Nephrotoxicity assessment}

Cisplatin (Sigma Aldrich) was applied to the top compartment of confluent monocultures of hRPTEC and to cocultures with HUVEC. Cisplatin of 0, 50, 100 and $150 \mu \mathrm{M}$ in REGM complete medium was added to the inserts after 7 days of cell culture. The range of values was selected according to previously published papers which also performed nephrotoxicity assays, ${ }^{30,34}$ with the main aim of evaluating cell response to the increasing concentration of cisplatin. Cellular viability was assessed by alamarBlue as previously described and presented as a percentage of control viability (the inserts that were subjected to $0 \mu \mathrm{M}$ of cisplatin). To further evaluate cisplatin effect, live/dead assay with Calcein AM and propidium iodide (PI, both Sigma Aldrich) and immunofluorescence of DAPI and Phalloidin (ThermoFisher) were performed. The fluorescent dyes were incubated with the constructs at a concentration of $1 \mu \mathrm{g} / \mathrm{mL}$ for PI and $2 \mu \mathrm{g} /$ $\mathrm{mL}$ for Calcein AM in DPBS for 30 minutes at $37^{\circ} \mathrm{C}$. Cell fixation and immunofluorescence staining were performed as previously described. The cell-loaded membranes were visualized using a confocal microscope (above mentioned).

\subsection{Statistical analysis}

Statistical analyses were performed with GraphPad Prism 8 (GraphPad Software, California, USA). Data were expressed as means \pm standard deviation of experiments. Differences between groups were analysed by unpaired $t$ test or two-way analysis of variance (ANOVA) in case of experiments conducted over time, using Tukey test for post hoc assessments of the differences between samples. Statistical significance was defined as $P<.05$.

\section{ACKNOWLEDGEMENTS}

The authors want to acknowledge the financial support obtained by the European Regional Development Fund (ERDF) on the project FROnTHERA (NORTE-01-0145-FEDER-000023) and the FCT PhD Grant on the Doctoral Program on Advanced Therapies for Health (PATH) (PD/BD/128102/2016).

\section{CONFLICTS OF INTEREST}

None.

\section{ORCID}

Rita Sobreiro-Almeida (D) https://orcid.org/0000-0003-4433-8890 Maria Elena Melica (D) https://orcid.org/0000-0002-4599-4083 Laura Lasagni (iD https://orcid.org/0000-0001-6553-9160 Paola Romagnani (D) https://orcid.org/0000-0002-1774-8088 Nuno M. Neves (D) https://orcid.org/0000-0003-3041-0687

\section{REFERENCES}

1. ScottRP, QuagginSE. The cell biology of renal filtration. J Cell Biol. 2015;209(2):199-210.

2. KirkA, CampbellS, BassP, MasonJ, CollinsJ. Differential expression of claudin tight junction proteins in the human cortical nephron. Nephrol Dial Transplant. 2010;25(7):2107-2119.

3. SunC-Y, ChangS-C, WuM-S. Uremic toxins induce kidney fibrosis by activating intrarenal renin-angiotensin-aldosterone system associated epithelial-to-mesenchymal transition. PLOS ONE. 2012;7(3):e34026.

4. MasereeuwR, MutsaersHAM, ToyoharaT, et al. The Kidney and Uremic Toxin Removal: Glomerulus or Tubule?Semin Nephrol. 2014;34(2):191-208.

5. ZhangH, TasnimF, YingJY, ZinkD. The impact of extracellular matrix coatings on the performance of human renal cells applied in bioartificial kidneys. Biomaterials. 2009;30(15):2899-2911.

6. PrangeJA, BieriM, SegererS, et al. Human proximal tubule cells form functional microtissues. Pflügers Arch - Eur J Physiol. 2016;468(4):739-750.

7. WeberEJ, ChapronA, ChapronBD, et al. Development of a microphysiological model of human kidney proximal tubule function. Kidney Int. 2016;90(3):627-637. 
8. WilmerMJ, $\mathrm{NgCP}$, LanzHL, VultoP, Suter-DickL, MasereeuwR. Kidney-on-a-chip technology for drug-induced nephrotoxicity screening. Trends Biotechnol. 2016;34(2):156-170.

9. JansenJ, De NapoliIE, FedecostanteM, et al. Human proximal tubule epithelial cells cultured on hollow fibers: living membranes that actively transport organic cations. Sci Rep. 2015;5(1):16702.

10. AydinS, SignorelliS, LechleitnerT, et al. Influence of microvascular endothelial cells on transcriptional regulation of proximal tubular epithelial cells. Am J Physiol Physiol. 2008;294(2):C543-C554

11. LinasSL, RepineJE. Endothelial cells regulate proximal tubule epithelial cell sodium transport. Kidney Int. 1999;55(4):1251-1258.

12. KimB-S, ChenJ, WeinsteinT, NoiriE, GoligorskyMS. VEGF expression in hypoxia and hyperglycemia: reciprocal effect on branching angiogenesis in epithelial-endothelial co-cultures. J Am Soc Nephrol. 2002;13(8):2027-2036.

13. TasnimF, ZinkD. Cross talk between primary human renal tubular cells and endothelial cells in cocultures. Am J Physiol Physiol. 2012;302(8):F1055-F1062.

14. VedulaEM, AlonsoJL, ArnaoutMA, CharestJL. A microfluidic renal proximal tubule with active reabsorptive function. PLoS ONE. 2017;12(10):e0184330.

15. SakolishCM, MahlerGJ. A novel microfluidic device to model the human proximal tubule and glomerulus. RSC Adv. 2017;7(8):4216-4225.

16. Sobreiro-AlmeidaR, FonsecaDR, NevesNM. Extracellular matrix electrospun membranes for mimicking natural renal filtration barriers. Mater Sci Eng C. 2019;103:109866.

17. SagrinatiC, NettiGS, MazzinghiB, et al. Isolation and characterization of multipotent progenitor cells from the Bowman's capsule of adult human kidneys. J Am Soc Nephrol. 2006;17(9):2443-2456.

18. RonconiE, SagrinatiC, AngelottiML, et al. Regeneration of glomerular podocytes by human renal progenitors. J Am Soc Nephrol. 2009;20(2):322-332.

19. AngelottiML, RonconiE, BalleriniL, et al. Characterization of renal progenitors committed toward tubular lineage and their regenerative potential in renal tubular injury. Stem Cells. 2012;30(8):1714-1725.

20. NagaoRJ, XuJ, LuoP, et al. Decellularized human kidney cortex hydrogels enhance kidney microvascular endothelial cell maturation and quiescence. Tissue Eng Part A. 2016;22(19-20):1140-1150.

21. HynesRO. Integrins: Versatility, modulation, and signaling in cell adhesion. Cell. 1992;69(1):11-25.

22. DenchaiA, TartariniD, MeleE. Cellular response to surface morphology: electrospinning and computational modeling. Front Bioeng Biotechnol. 2018;6:155. https://doi.org/10.3389/ fbioe.2018.00155.

23. ChenM, PatraPK, LovettML, KaplanDL, BhowmickS. Role of electrospun fibre diameter and corresponding specific surface area (SSA) on cell attachment. J Tissue Eng Regen Med. 2009;3(4):269279. https://doi.org/10.1002/term.163.
24. MolitorisBA, WagnerMC. Surface membrane polarity of proximal tubular cells: Alterations as a basis for malfunction. Kidney Int. 1996;49(6):1592-1597.

25. LinNYC, HomanKA, RobinsonSS, et al. Renal reabsorption in 3D vascularized proximal tubule models. Proc Natl Acad Sci U S A. 2019;116(12):5399-5404.

26. IshidaS, LeeJ, ThieleDJ, HerskowitzI. Uptake of the anticancer drug cisplatin mediated by the copper transporter Ctr1 in yeast and mammals. Proc Natl Acad Sci. 2002;99(22):14298-14302.

27. LudwigT, RiethmüllerC, GekleM, SchwerdtG, OberleithnerH. Nephrotoxicity of platinum complexes is related to basolateral organic cation transport. Kidney Int. 2004;66(1):196-202.

28. KingSM, HigginsJW, NinoCR, et al. 3D proximal tubule tissues recapitulate key aspects of renal physiology to enable nephrotoxicity testing. Front Physiol. 2017;8:123.

29. NguyenDLG, KingSM, PresnellSC. US20190041381 - Engineered Renal Tissues, Arrays Thereof, and Methods of Making the Same. 2019.

30. JangK-J, MehrAP, HamiltonGA, et al. Human kidney proximal tubule-on-a-chip for drug transport and nephrotoxicity assessment. Integr Biol. 2013;5(9):1119-1129.

31. ProzialeckWC, EdwardsJR, LamarPC, SmithCS. Epithelial barrier characteristics and expression of cell adhesion molecules in proximal tubule-derived cell lines commonly used for in vitro toxicity studies. Toxicol Vitr. 2006;20(6):942-953.

32. SalehiM, Naseri-NosarM, GhorbaniS, FarzamfarS, AzamiM. Wetelectrospun PCL/PLLA blend scaffolds: effects of versatile coagulation baths on physicochemical and biological properties of the scaffolds. Regeneration, Reconstruction \& Restoration. 2017;2(1):1-7.

33. LazzeriE, RonconiE, AngelottiML, et al. Human urine-derived renal progenitors for personalized modeling of genetic kidney disorders. J Am Soc Nephrol. 2015;26(8):1961-1974.

34. CzernieckiSM, CruzNM, HarderJL, et al. High-throughput screening enhances kidney organoid differentiation from human pluripotent stem cells and enables automated multidimensional phenotyping. Cell Stem Cell. 2018;22(6):929-940.e4.

\section{SUPPORTING INFORMATION}

Additional supporting information may be found online in the Supporting Information section.

How to cite this article: Sobreiro-Almeida R, Melica ME, Lasagni L, Romagnani P, Neves NM. Cocultures of renal progenitors and endothelial cells on kidney decellularized matrices replicate the renal tubular environment in vitro. Acta Physiol. 2020;230:e13491. https://doi.org/10.1111/apha.12527 